\title{
Tuning nanomechanical properties of
}

\section{microstructures made by 3D direct laser writing}

Mehdi Belqat ${ }^{1,2}$, Xingyu $W^{1,2}$, Laura Piedad Chia Gomez ${ }^{1,2}$, Jean-Pierre Malval ${ }^{1,2}$, Sébastien 
ABSTRACT

3D Direct laser writing (3D DLW) based on two photon polymerization represents a powerful technique for the additive manufacturing of 3D micro- and nano-structures, which have a number of promising applications in areas including biology and cell cultures up to soft robotic. New applications often entail the contact and movement of nanoscale areas, therefore understanding of the mechanical properties involved in such systems is necessary for creating reliable micro and nanoscale applications. Notably, knowledge about nanomechanics of 3D printed structures and its dependence on its geometry and contact size remains essential. This study focuses on the investigation of the Young's modulus of 3D printed microstructures by Atomic Force Microscopy. Using the PeakForce QNM AFM mode, it was possible to determine the influence of the laser power and the monomer and their mixture on the micromechanical properties, in particular the Young's modulus. The fabricated structures are 2D structures, consisting of successive lines forming a square. Three different resins have been used based on poly(ethyleneglycol)diacrylate (PEGDA), trimethylolpropane triacrylate (TMPTA) and pentaerythritol triacrylate (PETA). The results obtained show that the increase in laser power increases the Young's modulus of these raw materials, since it induces a stronger crosslinking density. Mixtures of resins have also been formulated inducing a Young's modulus structure increase when increasing the amount of PETA. By combining these two approaches, tuning of the nanomechanical properties of the final microstructures on an unprecedented range covering 3 orders of magnitude, from MPa up to GPa have been successfully achieved. This major result paves the way to in-depth reflection on future works in nanorobotics or biomedical devices, where complex mechanical behavior are highly desired. 


\section{Introduction}

54 Characterization of mechanical properties of structures and materials are of primary importance for their use in a broad range of applications. The first known scientific research on the mechanics of building elements dates back only to the end of the $16^{\text {th }}$ century with the famous work of Galileo on the tension and bending of beams. But, science has evolved and last century has brought new characterization techniques that have been developed to face it. In parallel, new generation of materials and complex composites have also been created and built at different scales. Among the various fabrication techniques available to shape the material, 3D direct laser writing (3D DLW) based on two photon real damage in its bulk or on its surface.

Nevertheless, measuring mechanical properties of micro-objects via conventional methods is impossible polymerization represents a powerful technique for the fabrication of 3D micro- and nano-structures [13]. This technique allows to perfectly replicate miniaturized 3D objects, process that could not be achieved with traditional microfabrication technologies such as self-assembly technique [4], electron beam lithography [5] and nanoimprint lithography [6]. In addition, physico-chemical and mechanical properties of these microstructures can be easily tuned by varying different technical parameters such as nature of resins, laser power and writing speed [7-9]. However, the characterization of such microobjects remains difficult, while the control of the mechanical properties of these microstructures is essential for their future applications. Indeed, the measurement of the Young's modulus of microobjects is crucial to achieve the microstructures with a high fidelity. For instance, in bioengineering, tuning rigidity of a scaffold or a prothesis allows to enhance the biocompatibility of the objects in its new environment [10]. In addition, the detection of a mechanical defect on a microstructure can avoid a due to the size of these objects. In order to circumvent these limitations, several characterization techniques have been proposed and developed to study the mechanical properties of micro and nanostructures. Treacy et al. [11] have estimated the Young's modulus of isolated carbon nanotubes by measuring, in transmission electron microscopy, the amplitude of their intrinsic thermal vibration. This original method, although highly powerful, can only be used for specific and conductive micromaterials 
such as carbon nanotubes. The three-point bending test is commonly used to test the bending strength of materials. Inspired from this principle, Lemma et al. [12] have proposed a bending characterization method of the mechanical properties of microstructures produced by two-photon stereolithography. They have combined static and dynamic mechanical analysis on pillar-like structures and picometersensitive laser Doppler vibrometry of drum-like structures to viably and nondestructively estimate Young's modulus, Poisson's ratio, and density of 3D printed micromaterials. This innovative and nondestructive method can however only be used for microstructures having a specific shape. Shin et al. [13] have proposed another method to induce microbending in microstructures and to determine their Young's modulus. For this, microcantilever bars were manufactured and at the end of these structures, a microsphere was fabricated in order to induce bending using optical tweezers. The sphere was then trapped by an optical beam and was able to move over several microns depending on the strength of the optical trap. Young's modulus of the microcantilever could be indirectly estimated from the deviation of the bar during the optical stress. Other destructive techniques such as in situ mechanical test in scanning electronic microscope could have been used to estimate mechanical properties. Geer et al. [14] have measured deformation of fractal-like architectures by micro-compression test and developed a computational model that was able to capture local stress distributions within the nanolattices under this solicitation. As a result, they could explain some of the underlying deformation mechanisms and validate the measured effective stiffness of the metamaterial such as for 3D microprinted Eiffel Tower. Finally, micro and nanoindentation remains one of the most commonly used tools independently of its destructive character. The principal goal of a nanoindentation test is to extract elastic modulus and hardness of a specimen material from experimental readings of the indenter load versus the depth of penetration. Lee et al. [15] have used the microindentation technique to determine the Young's modulus and compressive strength of different architectures (square, pillars and metamaterials) at the micrometric scale. In a different way, Oakdale et al. [16] sought to assess the influence of density as well as the influence of UV post-polymerization on Young's modulus by conducting compression tests on Woodpiles using a nanoindenter. Young's moduli were extracted from the slope of the load curves. Bauer et al. [17] did similar compressive and destructive tests at microscale on pyrolyzed resins to 
determine the influence of the size of carbon nanolattices as well as the influence of an alumina coating

107 on the Young's modulus of the 3D-printed microstructures. Lastly, Barner-Kowollik et al. [18] have 108 used nanodynamical mechanical analysis (NanoDMA) to investigate the bulk viscoelastic properties 109 (hardness, complex elastic modulus, and loss factor) of the microstructures over the course of irradiation 110 of polymer at the nanoscale. This technique is a quite new and powerful technology to characterize the 111 bulky mechanical properties of microstructures produced by 3D DLW. In a complementary approach, 112 Atomic Force Microscopy in PeakForce QNM mode, which also represents an impressive and non113 destructive test, allows determining mechanical properties of microstructure surface. Gou et al. [19] 114 have investigated the mechanical properties of hydrogels produced by two-photon stereolithography 115 using atomic force microscopy. In the Quantitative Nanomechanical Property Mapping (QNM) mode, 116 they were able to determine the influence of the manufacturing laser power on the Young's modulus on 117 their different 3D printed samples. All these different examples have shown that new generation of 118 technics have been developed to allow determining the intrinsic mechanical properties of 3D printed 119 microstructures depending on their geometry, nature and size. Thus, the knowledge of fundamental 120 nanomechanics mechanism of these micromaterials remains essential. Even though the stakes are so 121 high regarding the targeting applications, the influence of the fabrication parameters on mechanical 122 properties are not systematically investigated when designing new advanced materials.

123 In this study, we have proposed to investigate three different photosensitive resins, PEGDA

124 (Poly(ethyleneglycol)diacrylate), Trimethylolpropane Triacrylate (TMPTA) and PETA (Pentaerythritol 125 triacrylate) to create microstructures of varying stiffness by 3D DLW. The apparent Young moduli of 126 these materials have been determined by AFM in PeakForce QNM. Two parameters such as the 127 increase of the laser power and the type of resins mixture have allowed to tune the nanomechanical 128 properties of the final microstructures on a large range, from MPa up to GPa. These results pave the 129 way to in-depth reflection on future works in nanorobotics or cell biology, where complex mechanical 130 behavior are highly desired.

\section{Materials and Methods}


133 Pentaerythritol triacrylate (PETA) $(\rho=1,18 \mathrm{~g} / \mathrm{ml} ; \mathrm{M}=298,29 \mathrm{~g} / \mathrm{mol})$ and Poly(ethylene Glycol)

134 diacrylate PEGDA ( $\rho=1,12 \mathrm{~g} / \mathrm{ml} ; \mathrm{M}=700 \mathrm{~g} / \mathrm{mol}$ ) were purchased from Sigma-Aldrich. Diphenyl

135 (2,4,6- trimethylbenzoyl)- phosphine oxide (TPO-L) was purchased from BASF (M = 336,371 g/mol).

136 Trimethylolpropane Triacrylate (TMPTA) was kindly provided by Sartomer $(\rho=1,1 \mathrm{~g} / \mathrm{ml} ; \mathrm{M}=296,32$ $137 \mathrm{~g} / \mathrm{mol}$ ). The chemical formula of all the products are depicted in Figure 1.

a)<smiles>C=CC(=O)OCC(CO)(COC(=O)C=C)COC(=O)C=C</smiles>

c)

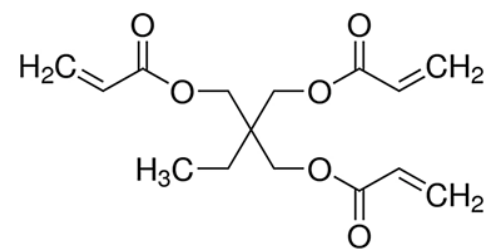

b)<smiles>C=CC(=O)OCCOC(C)=O</smiles>

d)<smiles>CCOP(=O)(C(=O)c1c(C)cc(C)cc1C)c1ccccc1</smiles>

Figure 1. Chemical formula : a) PETA; b) PEGDA ; c) TMPTA ; d) TPO-L.

\subsection{Characterization}

\subsubsection{Microfabrication by 3D DLW}

142 A detailed description of the 3D microfabrication set-up has been previously described elsewhere. [8, 143 20] However for the sake of clarity, it has to be mentionned that the generation of microstructures has 144 been done via the computer-aided design (CAD) module of Simpoly allowing the control of specific 145 parameters such as the percentage of overlap $\Delta_{\mathrm{V}}$ between each voxel or the distance $\Delta_{\mathrm{l}}$ between two lines 146 forming the microstructure (Figure 2a). The two photon excitation was performed at $800 \mathrm{~nm}$ by 147 focusing the femtosecond laser beam with an objective lens (x40, NA: 0.65) into a drop of resist (Figure 148 2b). This substrate was previously functionalized with 3-(triethoxysilyl) propyl methacrylate to enhance 149 the adhesion of the structures onto the substrate. The 3D microstructure is finally obtained by washing 150 away the unreacted monomer using ethanol (Figure 2c). 
a)

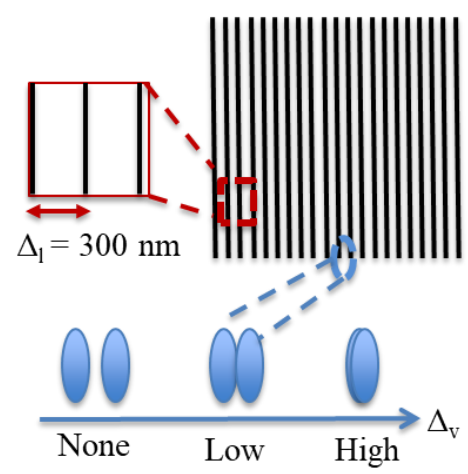

b)

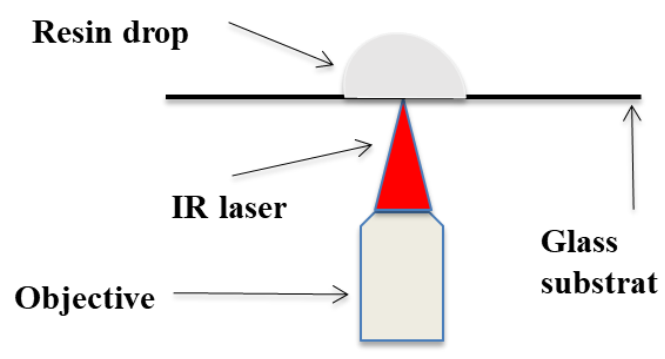

c)

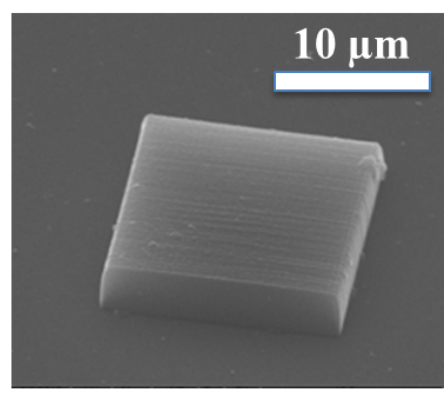

Figure 2. 3D DLW fabrication steps of a microstructure. a) CAD modelization and writing strategy for square microstructure, b) Photofabrication based on two photon polymerization, c) SEM image of typical microstructure obtained after microprinting.

\subsubsection{Atomic Force Microscopy}

The investigation of the morphology, adhesives and mechanical properties of all the 3D microstructures have been performed by Atomic Force Microscope (AFM). A Bruker Multimode 8 AFM in Peakforce Quantitative Nanomechanics (PeakForce QNM) mode has been used to study the physico-chemical and mechanical nanoscale features of the 3D microstructures. Particularly, PeakForce QNM mode is a recent advanced mode developed by Bruker to investigate quantitative nanomechanical mapping properties with the simultaneous measurement of the sample's adhesion between tip and sample surface, Young's modulus (according to either Derjaguin-Muler-Toporov (DMT) or Sneddon model), deformation and energy dissipation along with the surface topography [21]. The imaging scan size was $(5 \mu \mathrm{m} \times 5 \mu \mathrm{m})$. The physico-chemical parameters average such as roughness (RMS), adhesion and spell out this abbreviation (DMT) Module were determined using the Gwydion software. QNM etched silicon probes were provided by Bruker. They were RTESPA-150 presenting a nominal spring constant $\mathrm{k} \sim 6 \mathrm{~N} / \mathrm{m}$ and RTESPA-300 presenting a nominal spring constant $\mathrm{k} \sim 40 \mathrm{~N} / \mathrm{m}$. All used tips were calibrated according to Bruker's user guide [21] and norm ISO13095:2014. The test operation steps of relative method in

170 PeakForce QNM were described as followed: firstly, a Sapphire-15M (supplied by Bruker) was used for 171 calibration of deflection sensitivity of cantilever. Then, the stiffness $\mathrm{k}$ of the cantilever was calibrated according to thermal noise method. Secondly, the $\mathrm{k}$ and $\mathrm{R}$ values were adjusted to make the acquired 
173

174

175

176

177

178

179

180

181

182

183

184

185

186

modulus consistent with the standard values obtained with calibration samples (supplied by Bruker).

Thirdly, the sample was carried out. The results were analysed by both Nanoscope analysis 2.0 software provide by Bruker and Gwyddion. The root mean square (RMS) roughness, adhesion and DMT modulus parameters were measured for at least three representative AFM images from different areas of 3D microstructures, and at least three different samples for each film were studied and analysed as previously described in S.Oras et al. [22]. The Derjaguin-Muler-Toporov (DMT) model can be viewed as a modified Hertzian model, which takes into account the adhesive forces between the tip and the surface. According to this approach, the reduced Young's modulus, Er, is given by [23].

$$
E_{r}=\frac{3\left(F_{t i p}-F_{a d h}\right)}{4 \sqrt{R d^{3}}}
$$

In Eq. (1), Ftip is the force on the AFM tip, Fadh is the adhesive force between the AFM tip and sample, $\mathrm{R}$ is the AFM tip radius, and $\mathrm{d}$ is the deformation depth. The reduced Young's modulus is related to the sample Young's modulus (Es) and is calculated by Eq. (2) [15].

$$
\frac{1}{E_{r}}=\frac{\left(1-v_{S}^{2}\right)}{E_{S}}+\frac{\left(1-v_{I}^{2}\right)}{E_{I}}
$$

where $\mathrm{E}_{\mathrm{I}}$ is the indenter Young's modulus, $v_{\mathrm{I}}$ is the Poisson's ratio of the indenter and $v_{\mathrm{S}}$ is Poisson's ratio of the sample.

\subsubsection{Fourier-Transform Infrared (FTIR) microspectroscopy}

The investigation of the degree of conversion (DC) has been performed by FTIR microspectroscopy using a Thermo Scientific Nicolet iN10 MX equipped with a germanium tip for the ATR mode. Typically, the analyzed zone was 5 micrometers at the center of squared polymeric structures of 20 micrometers length. The absorbance spectra were measured from $4000 \mathrm{~cm}^{-1}$ to $675 \mathrm{~cm}^{-1}$ wavenumber with a resolution of $8 \mathrm{~cm}^{-1}$ and 64 scans per spectrum. This method relies on measuring the degree of conversion (DC) of the structured polymers in comparison with the resin mixture and is determined by Equation (3) [24]: 


$$
D C=\left[1-\frac{A_{C=C} / A_{C=O}}{A^{\prime} C=C / A^{\prime} C=O}\right] \times 100
$$

198 where $\mathrm{A}_{\mathrm{C}=\mathrm{C}}, \mathrm{A}_{\mathrm{C}=\mathrm{O}}, \mathrm{A}^{\prime}{ }_{\mathrm{C}=\mathrm{C}}$ and $\mathrm{A}^{\prime} \mathrm{C}=\mathrm{O}$ are the integrated area of absorption bands related to the $\mathrm{C}=\mathrm{C}$ and

$199 \mathrm{C}=\mathrm{O}$ moieties in the polymerized and the nonpolymerized resin, respectively.

\section{$200 \quad$ Results and discussion}

201 The size and the structure of the samples have been first optimized before investigating the influence of

202 the different parameters such as the laser power and nature of the resin on the nanomechanical

203 properties of the 3D microstructures. Indeed, it was first necessary to determine the overlap between two 204 consecutive voxels and two adjacent lines on the surface in order to minimize the roughness of the 205 sample, which might also induce an impact on the modulus and adhesion measurements. Besides, the

$2063 \mathrm{D}$ architecture itself and the way photopolymerization is carried out may impact the mechanical 207 responses of the microstructure [25], therefore we have limited our study to squared microtructures 208 made of a single layer. The 3D DLW manufacturing of a sample starts by focusing a beam under a 209 microscope resulting on the point-by-point photopolymerization of a resin. This point, corresponding to 210 a voxel, takes the form of an ellipsoid and represents the building block of the fabricated structure. The

211 overlap $\left(\Delta_{\mathrm{v}}\right)$ characterizes the distance between the voxels, illustrated in Figure 2a. 
a)

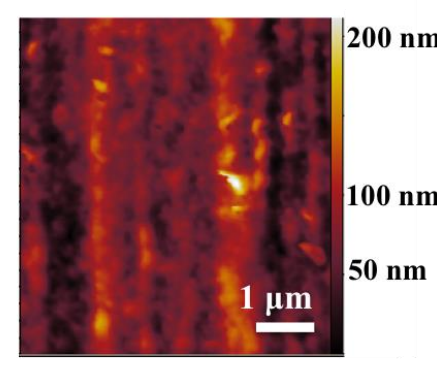

$\Delta_{\mathrm{l}}=100 \mathrm{~nm}$ b)

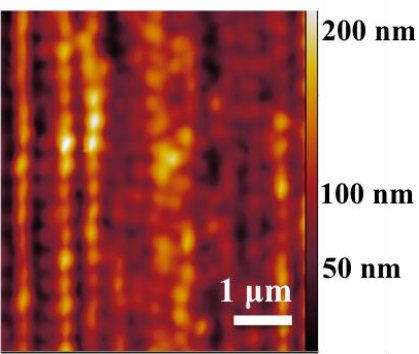

$\Delta_{\mathrm{l}}=200 \mathrm{~nm}$ c)

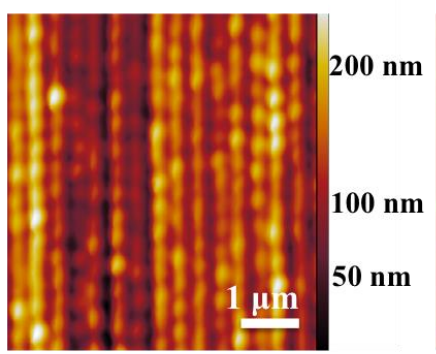

$\Delta_{\mathrm{l}}=300 \mathrm{~nm}$ d)

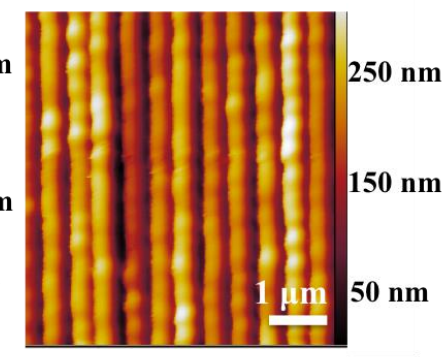

$\Delta_{\mathrm{l}}=400 \mathrm{~nm}$

e)

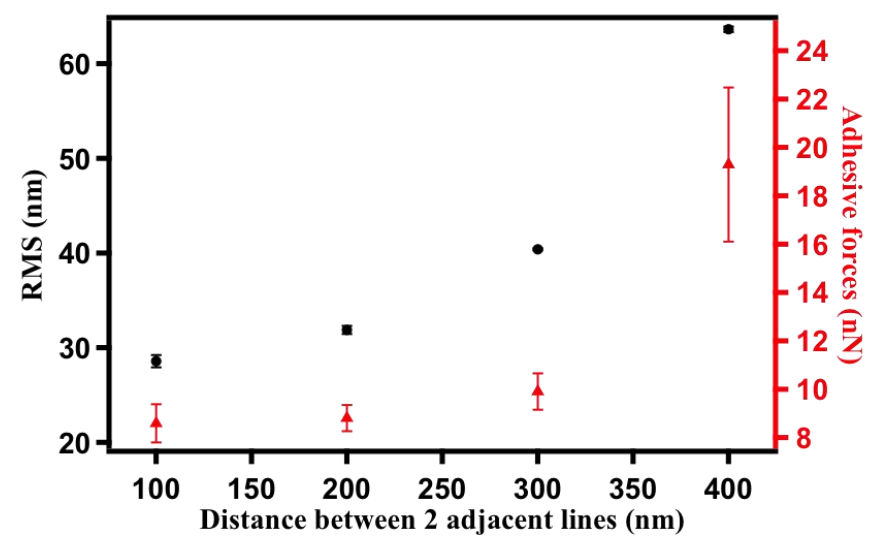

213 Figure 3. a-d) AFM topography images of PETA 25x25 $\mu \mathrm{m}^{2}$ square samples (PeakForce QNM mode)

214 varying as function of overlap parameter. e) Graph depicting the evolution of roughness (RMS)-(black 215 point) and adhesive forces (red triangle) as function of layer distance $\left(\Delta_{1}\right)$.

217 For a given line, this overlap parameter between two successive voxels was set at $60 \%$ in the CAD

218 software, allowing a low roughness in the writing direction of the sample as depicted in Figure $3 \mathrm{~d}$.

219 The overlap $\left(\Delta_{1}\right)$ characterizes the distance between two adjacent lines (Figure $\left.2 \mathrm{a}\right)$. As expected, the 220 lower the overlap is, the higher the roughness is (Figure 3). However, in case of high overlap (i.e. low 221 distance between two adjacent lines), the difference between the threshold energy and the damage 222 energy, defining the possible manufacturing window, is greatly reduced. For sake of clarity, the 223 threshold energy represents the minimum energy required by the system to initiate a

224 photopolymerization phenomenon, and the damage energy corresponds to the energy leading to the 225 sample degradation. Besides, reducing the overlap $\left(\Delta_{1}\right)$ can significantly increase the manufacturing 226 time. For instance, $25 \times 25 \mu \mathrm{m}^{2}$ square microstructures used for mechanical investigations are fabricated in $12 \mathrm{~min}$ or $3 \mathrm{~min}$ respectively for $\Delta_{1}=100 \mathrm{~nm}$ or for $\Delta_{1}=400 \mathrm{~nm}$. In order to optimize the fabrication 
228 parameters, different overlap values between two adjacent lines, have been tested and the roughness of 229 the average roughness type (Root Mean Square-RMS) and the forces of adhesion were measured by 230 AFM as observed in Figure 3. The graph in Figure 3e shows that roughness increases with the distance 231 value between two adjacent lines, as theoretically expected. Adhesion force also slowly increases with 232 this parameter, however the overall variation of the value remains weak. The latter can be explained by 233 the fact that, when the overlap is low, lines are separated and acts as single units instead of being stick 234 and/or polymerized with the adjacent ones. As a result, it increases roughness of the overall surface, and 235 it also leads to heterogeneity in chemical properties as illustrated by the evolution of error bars for 236 adhesive forces. Finally, for $\Delta_{1}=300 \mathrm{~nm}$, the roughness has been also characterized in function of the 237 average laser power and the nature of the resists used (Figures S1 to S5, and Tables S3 to S6). While for 238 a given monomer, no trends of the RMS have been noticed in function of the laser power, one can 239 observed a slightly higher RMS values for TMPTA $(50 \mathrm{~nm})$ and PETA $(41 \mathrm{~nm})$ compare to PEGDA (27 $240 \mathrm{~nm}$ ). Additionally, SEM images provided in supporting information confirm the low impact of 241 aforementioned parameters on the surface morphology of the 2D microstructures (Figures S6, S7 and 242 S8). Consequently, a distance of $300 \mathrm{~nm}$ between lines has been fixed in the mechanical studies 243 described hereafter.

244 Then, three different resins (PEGDA, TMPTA, PETA) have been photopolymerized using previous 245 conditions to assess the evolution of the square sample topography as well as their mechanical 246 properties (variation of their Young's modulus). Their corresponding approach-retract curves have been 247 also presented in Figure 4. 
a1)
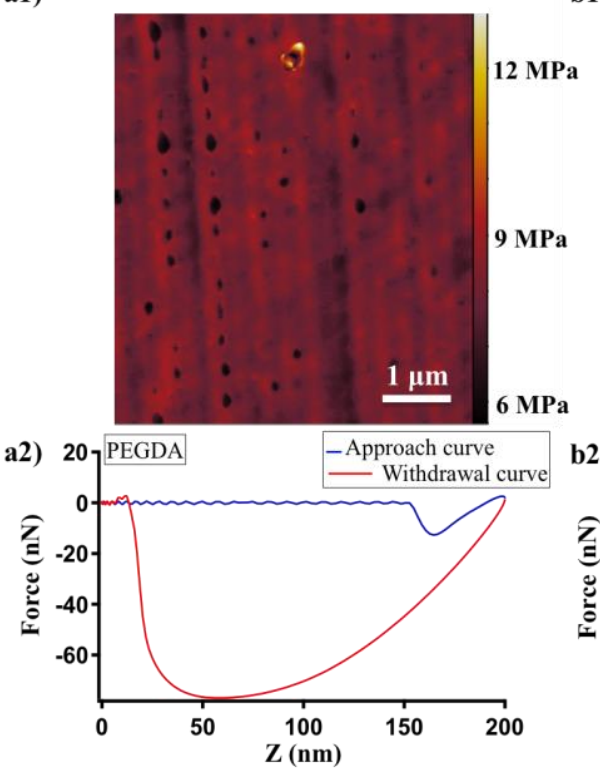

b1)
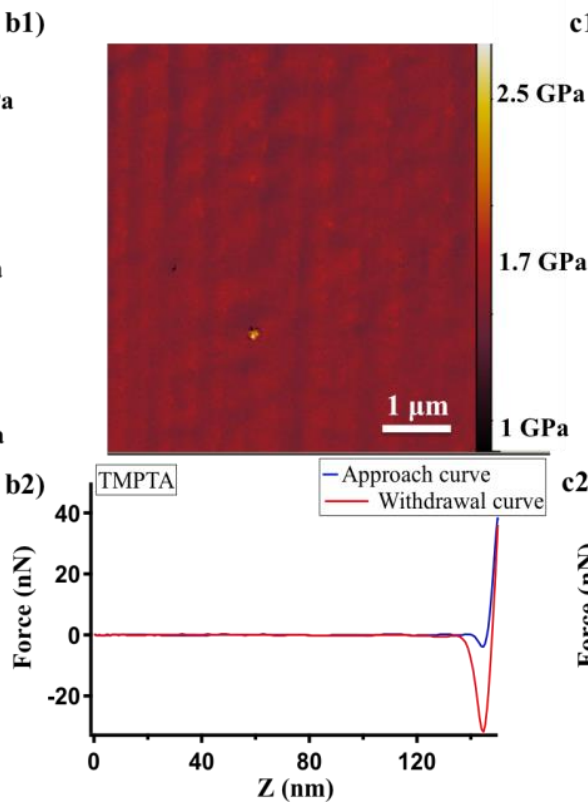

c1)
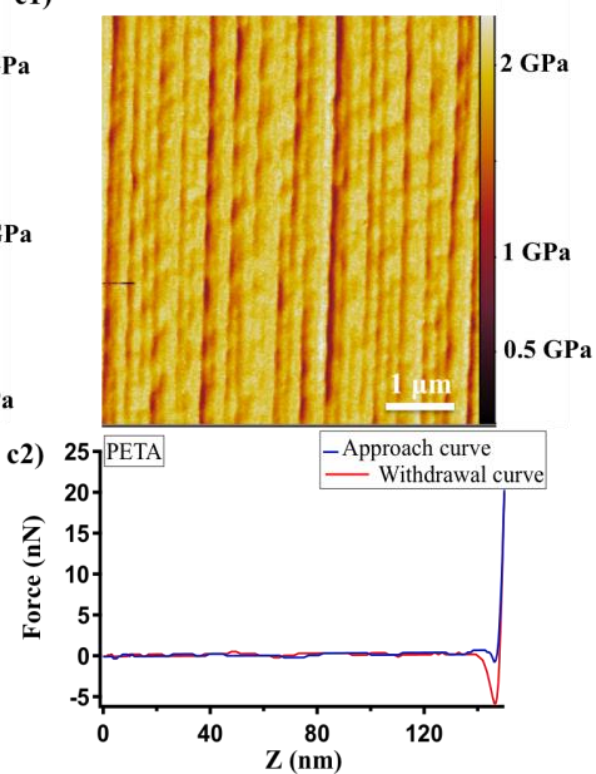

Figure 4. AFM topographic images in PeakForce QNM mode and their corresponding approach-retract curves: a1-2) PEGDA; b1-2) TMPTA; c1-2) PETA.

The approach-retract curves in Figure 4 a2, b2, c2 are formed by the superposition of a blue curve which corresponds to the approach curve and of a red curve corresponding to the withdrawal curve of the tip from the surface. The difference between these two curves corresponds to the energy dissipated by the tip during contact. This Hysteresis is higher for a soft material (Figure 4-a2), and smaller for a rigid material as observed in Figure 4-c2. The Young's modulus corresponding to the three different resins with distinctive processable parameters are summarized in Table 1. 
Table 1. Young's Modulus obtained for the three different monomers PEDA, TMPTA and PETA photopolymerized (manufactured with an $\mathrm{x} 40$ objective N.A. 0,65) for a laser power of $9.9 \mathrm{~mW}$, an exposure time of $10 \mathrm{~ms}$ and an overlap $\Delta_{1}=300 \mathrm{~nm}$, characterized by AFM in PF QNM mode.

\begin{tabular}{c|c|c|c} 
Monomers & DMT Young's & Cantilever's spring & Tip radius \\
& Modulus (MPa) & constant (N/m) & $(\mathbf{n m})$ \\
\hline PEGDA & $6,9+/-0,1$ & 6 & 80 \\
\hline TMPTA & $1669+/-56$ & 40 & 100 \\
\hline PETA & $1956+/-13$ & 40 & 100
\end{tabular}

These results are in good agreement with literature, in particular for PEGDA [19] and PETA [12]. TMPTA and PETA have similar Young's modulus value, this can be explained by the fact that TMPTA has a similar chemical structure to PETA, as a result both resins moduli are expected to be of the same order of magnitude. In 3D DLW process, two-photon polymerization (2PP) occurs in a very limited time and space scales compare to conventional photopolymerization. Thus, additionally to oxygen inhibition, dark polymerization or functionality of monomers, many other complex phenomenon such as optical aberration or local heating have to be taken into consideration in the formation of the polymer network [26]. However, as described by E. Andrzejewska [27], the process of network formation for free radical photopolymerization is strongly linked to the kinetics of photopolymerization. The functionality, defined here as the number of polymerizable groups per molecule, play a crucial role both on the kinetics and on the formation of higher cross-link density. Indeed, higher functionality involves higher concentration of functional groups which in turn leads to a faster formation of denser network. Therefore, one can expect higher cross-link density, and consequently higher Young's modulus in PETA and TMPTA compare to PEGDA as observed in Figure 4. Once the system was calibrated, it was possible to assess the influence of the laser power as well as that of the monomer mixture on the resin Young's modulus. 
288 The PETA, TMPTA and PEGDA samples were generated under the same conditions with an overlap $\Delta_{1}$ $289=300 \mathrm{~nm}$. The results obtained are presented in Figure 5.

a)

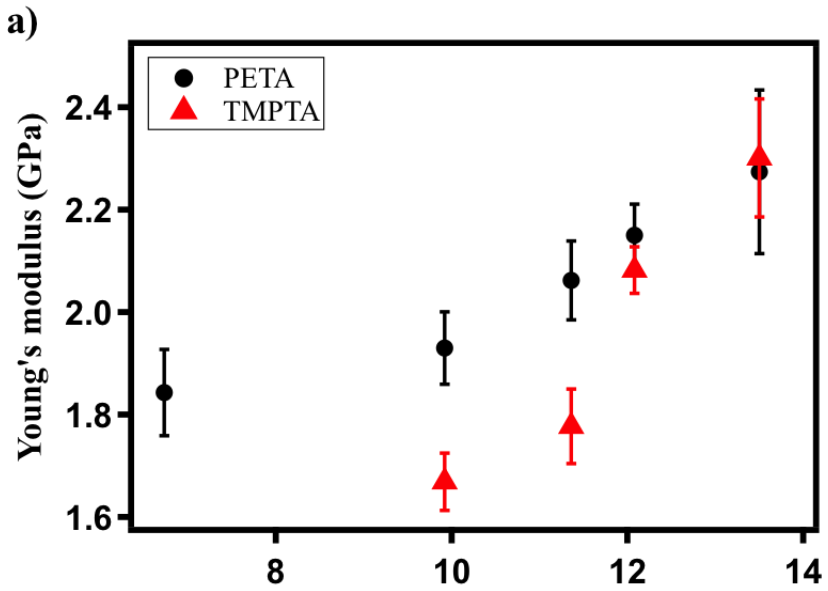

Average laser power (mW) b)

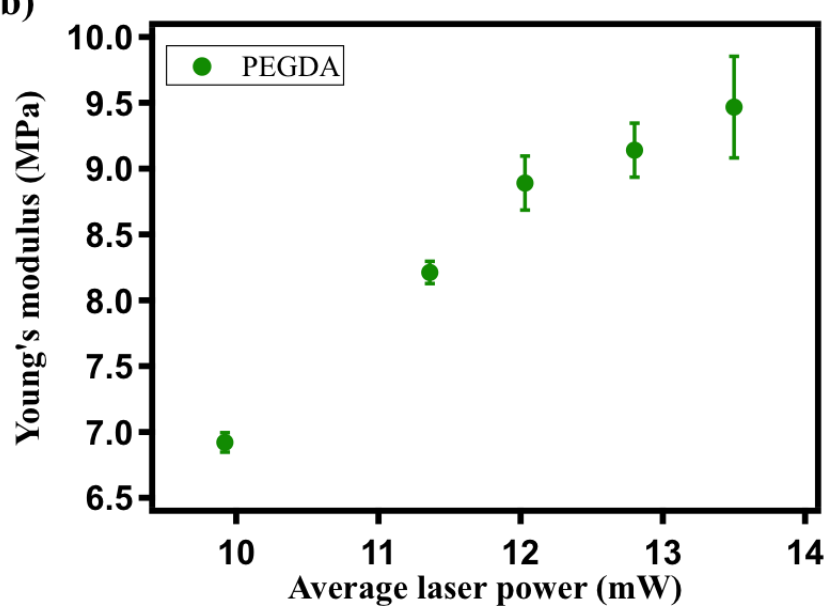

Figure 5. Evolution of Young's modulus as function of laser power for a) PETA (black circles) and TMPTA (red triangles), b) PEGDA (green circles).

From a general point of view, an increase in Young's modulus is observed as a function of writing laser power independantly of the nature of the monomer. For PETA, Young's modulus varies from 1.85 GPa for a power of $6.73 \mathrm{~mW}$ up to $2.27 \mathrm{GPa}$ for a power of $13.5 \mathrm{~mW}$ (Figure 5a). Such behavior is expected since the size of an individual voxel and the corresponding mechanical properties of the material are directly related to the energy of the laser pulse and the duration of exposure. Lu et al. [24] demonstrated a direct link between the laser power and the degree of conversion and therefore, by extension, the crosslinking density. Previous analyzes have shown that crosslink density is directly related to modulus of elasticity and ultimate tensile strength of crosslinked acrylic materials [28]. By comparison, Lemma et al. [12] observed an increase in Young's modulus of $1.5 \mathrm{GPa}$ for a power of $8.5 \mathrm{~mW}$ at $2 \mathrm{GPa}$ at 16 $\mathrm{mW}$, measured by micro-bending on an IP-L 780 resin (mainly composed of PETA monomer). The order of magnitude remains the same, however the values obtained by the latter are lower, which can be explained by different experimental conditions (numerical aperture of the microscope objective, overlap between each voxel, nature and concentration of photoinitiator, etc...). 
308 For TMPTA, the results obtained are presented in Figure 5a. A similar behavior as PETA has been 309 observed, with an increase in Young's modulus as a function of laser power. Indeed, Young's modulus 310 varies from $1.67 \mathrm{GPa}$ for a laser power of $9.9 \mathrm{~mW}$ to $2.3 \mathrm{GPa}$ for a power of $13.5 \mathrm{~mW}$. However, in 311 comparison with PETA, it seems that the increase is more significant (increase of $38 \%$ compared to 23 $312 \%$ for PETA). Chemically, both monomers have the same number of acrylic functions however, PETA 313 is more viscous $(500-1000 \mathrm{cP})$ than TMPTA $(80-135 \mathrm{cP})$ due to the possibility of forming hydrogen 314 bonds via the hydroxy group $(-\mathrm{OH})$. While it is known that initial viscosity of acrylate based resist can 315 drastically enhanced the reactivity of a given resists [29], further investigation will be necessary to state 316 on the role of viscosity on mechanical properties. More broadly, the viscosity has been scarcely 317 investigated in the frame of a two-photon polymerization as recently reported by Zandrini and 318 coworkers [30].

319 Finally, the evolution of mechanical properties of the PEGDA is displayed in Figure 5b. An increase in 320 the Young's modulus is observed as a function of the laser power, the Young's modulus varied from 6.9 $321 \mathrm{MPa}$ for a power of $9.9 \mathrm{~mW}$ to $9.5 \mathrm{MPa}$ for a power of $13.5 \mathrm{~mW}$, constituting an increase of $38 \%$. The 322 lower modulus values can be explained by the presence of only two acrylate functions for PEGDA 323 instead of three for PETA or TMPTA. Thus, a looser crosslinking network can be generally observed for 324 mono- or difunctional monomers. Finally, unlike other studies where PEGDA is used as a hydrogel, it 325 should be noted that in our formulation, no solvent has been used, which explains values of the order of $326 \mathrm{MPa}$. In addition, in presence of water, photopolymerized materials can exhibit Young's moduli of the 327 order of a few hundred $\mathrm{kPa}$.

328 To conclude, the results obtained confirm the link between the final Young's modulus of the material 329 and the manufacturing laser power, but also the influence of viscosity and the chemical nature of 330 monomer on the mechanical properties of the material. 
336 In order to be able to modulate the mechanical properties of the microstructures over a wider range, several 337 mixtures of PETA and PEGDA have been prepared to assess the chemical influence on their Young's 338 modulus. The results obtained are summarized in the graph in Figure 6a.

a)

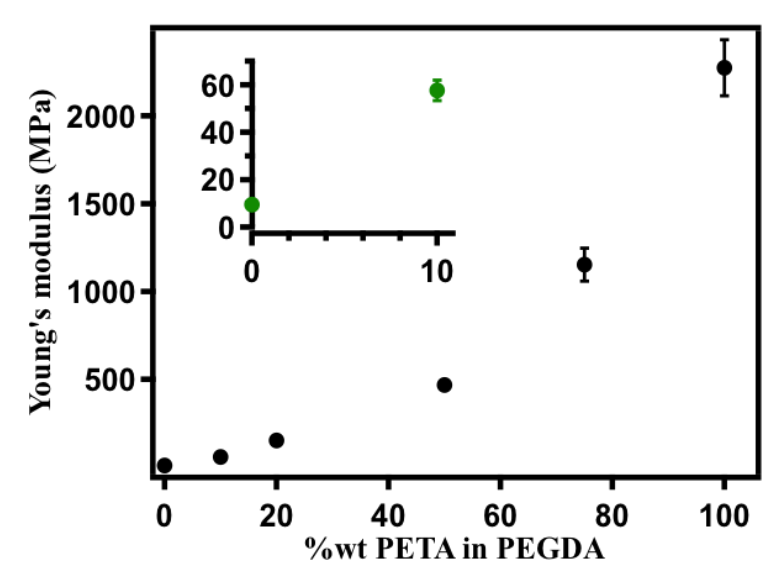

b)

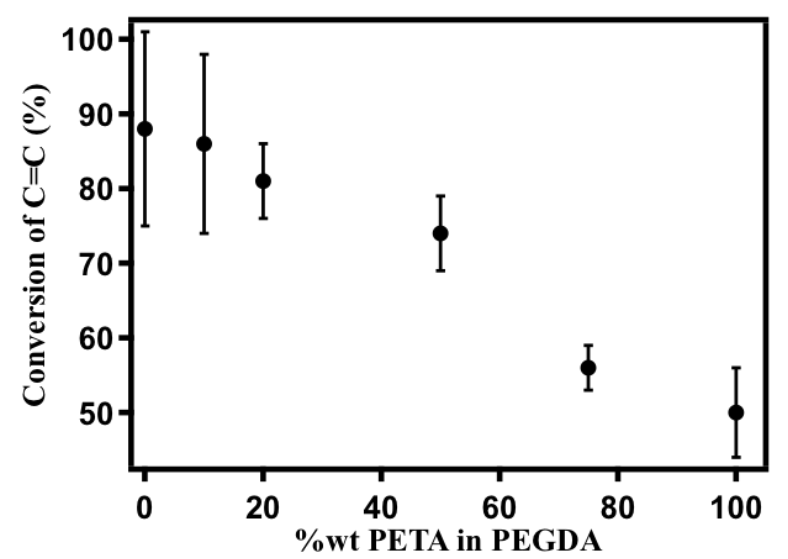

340 Figure 6. Evolution of a) Young's modulus and of b) degree of conversion as function of PETA 341 concentration in PEGDA for a laser power of $13.5 \mathrm{~mW}$, an exposure time of $10 \mathrm{~ms}$ and an overlap $\Delta_{1}=$ 342 300nm. The Young's moduli obtained for 0 and $10 \%$ of PETA in PEGDA have been highlighted in 343 inset (Green markers).

345 An increase in Young's modulus is observed as a function of the PETA concentration. Indeed, the 346 addition of $10 \%$ wt PETA induces an increase of the modulus from $9.4 \mathrm{MPa}$ up to $57 \mathrm{MPa}$. At $20 \%$ 347 PETA, the modulus reaches $150 \mathrm{MPa}$. For equal mixture, the Young's modulus increases to nearly 500 $348 \mathrm{MPa}$ and to $1.15 \mathrm{GPa}$, when the amount of PETA is equal to $75 \%$. On the other hand, Figure $6 \mathrm{~b}$ depicts 349 the evolution of the degree of conversion (DC) with the increase in PETA concentration in the final 350 mixture. The DC of polymerized microstructures have been determined using ATR-FTIR 351 microspectroscopy as proposed early by Cicha and coworkers [31]. Representative FTIR spectra for 352 different mixtures before and after polymerization are given in the supporting information (Figure S11). 353 Briefly, a value of $55 \%$ of conversion is determined in case of $100 \%$ of PETA. This result is in good 354 agreement with the work of Jiang et al. where a degree of conversion of $42 \%$ is determined by using 355 Raman microspectroscopy for a commercial resin, namely IP-L 780 which is mainly composed of 
PETA [24]. Besides, a value higher than $80 \%$ of conversion is observed for a microstructure made of

$357100 \%$ of PEGDA. Due to lack of sensitivity in our experimental set-up, we can not say with certainty

358 the absolute value, however the fact that this value is higher than the one reported for PETA is consistent with the fact that monomer of higher functionality leads to lower conversion [27]. Finally, contrary to what it has been noticed for mixture of monomers by Jiang et al. [32], when the

361 concentration of PETA increase from 0 to $100 \mathrm{wt} \%$ in the mixture, the degree of conversion is 362 progressively decreasing as expected. The latter highlights the impact of the monomer structure and 363 concentration on the final degree of conversion.

364 These overall results show that by tuning the mixture of two monomers, it has been possible to modulate 365 by 3 orders of magnitude (10 MPa, $100 \mathrm{MPa}$ and $1000 \mathrm{MPa})$, the mechanical properties of 3D 366 microstructures produced by 3D DLW, opening up new perspectives for the fabrication of devices with 367 complex behavior. In addition to those varied technical parameters, the writing speed remains also another key parameter that strongly impacts the Young's modulus of 3D microstructures, and which has also to be taken under consideration when designing new microdevices.

\section{Conclusion}

372 This research work deals with the need to finely control the mechanical properties of 3D micro-objects 373 produced by 3D DLW. Hence, evolution of the young's modulus of photopolymerized square microstructures has been investigated by AFM in PeakForce QNM mode, which represents an essential technique for the physico-chemical analysis of the surfaces of micrometric objects. Three main monomers commonly used in 3D DLW have been tested. Results have shown that it was possible to measure the apparent Young's moduli ranging from MPa to GPa at microscale. This parameter has 378 allowed to first assess the impact of photonic manufacturing parameters on the final mechanical properties of the material at microscale. Particularly, it appears that the increase in laser power induces 380 an increase in mechanical properties. Second, the influence of the monomer on the mechanical 381 properties has also been highlighted. It follows that the nature and structure of the monomer have a 382 direct influence on the Young's modulus. Third, it was possible to modulate over a wide range (3 orders 
383 of magnitude) the mechanical properties by acting on a mixture of monomers. This latter approach

opens up new perspectives in fields where complex deformations of the manufactured object are desired

385 (nanorobotics, nanosurgery) or where 3D spatial encoding of chemical and mechanical clues are highly

386 desired (cell biology, organ on chip, tissue engineering). Up to now, the variation of mechanical

387 properties remains moderate with regard to the challenges in terms of complexity required to develop

388 adaptive structures or multi-functional structures for the above mentioned applications such as

389 nanorobotics or cell biology. Thus, new routes should be kept in being explored.

\section{ACKNOWLEDGMENT}

394 The authors thanks the Agence Nationale de la recherche (Project ANR 2PhotonInsight: ANR-16-CE08395 0020) and Institut Carnot MICA for financial support. This work was also partially supported by an 396 Institutional Research Grant (MIPPI4D) from the Région Grand Est.

REFERENCES

[1] S. Maruo, O. Nakamura, S. Kawata, Three-dimensional microfabrication with two-photon-absorbed 401 photopolymerization. Opt. Lett. 1997, 2 (22), 132-134.

402 [2] C. N LaFratta, J. T Fourkas, T. Baldacchini,; R. A. Farrer, Multiphoton Fabrication. Angew. Chem., $403 \quad$ Int. Ed. 2007, 46, 6238-6258.

404 [3] C. Barner-Kowollik, M. Bastmeyer, E. Blasco, G. Delaittre, P. Müller, B. Richter, M. Wegener, 3D 405 Laser Micro- and Nanoprinting: Challenges for Chemistry. Angew. Chem., Int. Ed. 2017, 56 (50), 1582840615845.

407 [4] G.M. Whitesides, J.P. Mathias, C.T. Seto. Molecular Self-Assembly and Nanochemistry: A Chemical 408 Strategy for the Synthesis of Nanostructures. Science (80-. ). 1991, 254, 1312-1319.

409 [5] I. Haller, M. Hatzakis, R. Srinivasan. High-resolution Positive Resists for Electron-beam Exposure. 410 IBM J. Res. Dev. 1968, 12 (3), 251-256. 
411 [6] S.-W. Lee, K.-S. Lee, J. Ahn, et al. Highly Sensitive Biosensing Using Arrays of Plasmonic Au 412 Nanodisks Realized by Nanoimprint Lithography. ACS Nano 2011, 5 (2), 897-904.

413 [7] M. Hippler, E. Blasco, J. Qu, M. Tanaka, C. Barner-Kowollik, M. Wegener, M. Bastmeyer. 414 Controlling the shape of 3D microstructures by temperature and light. Nature Communications 2019, 10, 415232.

416 [8] L.P. Chia Gomez, A. Spangenberg, X.-A. Ton, Y. Fuchs, F. Bokeloh, J.-P. Malval, B. Tse Sum Bui, 417 D. Thuau, C. Ayela, K. Haupt, O. Soppera, Rapid prototyping of chemical microsensors based on 418 molecularly imprinted polymers synthesized by two-photon stereollithography. Adv. Mater. 2016, 28 (28), $419 \quad 5931-5937$.

420 [9] S.-Y. Yu, G. Schrodj, K. Mougin, J. Dentzer, J.-P. Malval, H.-W. Zan, O. Soppera, A. Spangenberg, 421 A. Direct Laser Writing of Crystallized TiO2 and TiO2 /Carbon Microstructures with Tunable Conductive 422 Properties. Adv. Mater. 2018, 30, 1805093.

423 [10] R. O’Rorke, T. Steele, H. Taylor. Bioinspired fibrillar adhesives: A review of analytical models and experimental evidence for adhesion enhancement by surface patterns. J. Adhes. Sci. Technol. 2015, 30. [11] M.M.J. Treacy, T.W. Ebbesen, J.M. Gibson. Exceptionally high Young’s modulus observed for individual carbon nanotubes. Nature. 1996, 381 (6584), 678-680.

[12] E.D. Lemma, F. Rizzi, T. Dattoma, et al. Mechanical Properties Tunability of Three-Dimensional 428 Polymeric Structures in Two-Photon Lithography. IEEE Trans. Nanotechnol. 2017, 16 (1), 23-31. [13] C.-S. Shin, T.-J. Li, C.-L. Lin. Alleviating Distortion and Improving the Young's Modulus in TwoPhoton Polymerization Fabrications. Micromachines. 2018, 9 (12), 615.

[14] L.R. Meza, A.J. Zelhofer, N. Clarke, et al. Resilient 3D hierarchical architected metamaterials. Proc.

433 [15] J.H. Lee, J.P. Singer, E.L. Thomas. Micro-/nanostructured mechanical metamaterials. Adv. Mater. $434 \quad 2012,24(36), 4782-4810$.

435 [16] J.S. Oakdale, J. Ye, W.L. Smith, J. Biener. Post-print UV curing method for improving the 436 mechanical properties of prototypes derived from two-photon lithography. Opt. Express. 2016, 24 (24), 437 27077. 
[17] J. Bauer, A. Schroer, R. Schwaiger, O. Kraft. Approaching theoretical strength in glassy carbon nanolattices. Nat. Mater. 2016, 15 (4), 438-443.

[18] M. Gernhardt, E. Blasco, M. Hippler, et al. Tailoring the Mechanical Properties of 3D 441 Microstructures Using Visible Light Post-Manufacturing. Adv. Mater. 2019, 31 (30), 1901269.

[19] X. Gou, M. Zheng, Y. Zhao, et al. Mechanical property of PEG hydrogel and the 3D red blood cell microstructures fabricated by two-photon polymerization. Appl. Surf. Sci. 2017, 416, 273-280. [20] M. Jin, J. Xie,a J-P. Malval, A. Spangenberg, O. Soppera, D-L. Versace, T. Leclerc, H. Pan, D. Wan, H. Pu, P. Baldeck, O. Poizat, S. Knopf. Two-photon lithography in visible and NIR ranges using multibranched-based sensitizers for efficient acid generation. J. Mater. Chem. C 2014, 2, 7201.

[21] Bruker PeakForce tapping brochure https://www.bruker.com/content/bruker/int/en/products-andsolutions/microscopes/materials-afm/afm-modes/peakforce-tapping.html

[22] S.Oras, S.Vlassov, M. Berholts, R. Lohmus, K. Mougin, Tuning adhesion forces between functionalized gold colloidal nanoparticles and silicon AFM tips: role of Ligands and capillary forces Beilstein J. Nanotechnol. 2018, 9, 660-670.

[23] B.. Derjaguin, V.. Muller, Y.. Toporov. Effect of contact deformations on the adhesion of particles.

J. Colloid Interface Sci. 1975, 53 (2), 314-326.

[24] L.J. Jiang, Y.S. Zhou, W. Xiong, et al. Two-photon polymerization: investigation of chemical and mechanical properties of resins using Raman microspectroscopy. Opt. Lett. 2014, 39 (10), 3034-3037. tuning of three-dimensional polymeric photonic crystals. Appl. Phys. Lett. 2007, 91, 241904.

458 [26] E. Skliutas, M. Lebedevaite, E. Kabouraki, T. Baldacchini, J. Ostrauskaite, M. Vamvakaki, M. 459 Farsari, S. Juodkazis, M. Malinauskas. Polymerization mechanisms initiated by spatio-temporally 460 confined light. Nanophotonics 2021; 10(4): 1211-1242

461 [27] E. Andrzejewska. Photopolymerization kinetics of multifunctional monomers. Progress in Polymer 462 Science, 2001, 26, 605-665. 
463 [28] T. Baldacchini, M. Zimmerley, C.-H. Kuo, E.O. Potma, R. Zadoyan. Characterization of

464 microstructures fabricated by two-photon polymerization using coherent anti-stokes Raman scattering 465 microscopy. J. Phys. Chem. B 2009, 113 (38), 12663-12668.

466 [29] A. Marcinkowska, E. Andrzejewska. Viscosity Effects in the Photopolymerization of Two467 Monomer Systems. Journal of Applied Polymer Science, 2010, 116, 280-287.

468 [30] T. Zandrini, N. Liaros, L.J. Jiang, Y.F. Lu, J.T. Fourkas, R. Osellame, T. Baldacchini. Effect of the 469 resin viscosity on the writing properties of two-photon polymerization. Optical Materials Express, 2019 $4709(6), 2601-2616$.

471 [31] K. Cicha, Z. Li, K. Stadlmann, A. Ovsianikov, R. Markut-Kohl, R. Liska, J. Stampfl. Evaluation of 4723 D structures fabricated with two-photon-photopolymerization by using FTIR spectroscopy. Journal of 473 Applied Physics, 2011, 110, 064911

474 [32] L. Jiang, W. Xiong, Y. Zhou, Y. Liu, X. Huang, D. Li, T. Baldacchini, L. Jiang, Y. Lu. 475 Performance comparison of acrylic and thiolacrylic resins in two-photon polymerization. Optics 476 Express, 2016, 24 (12), 13687-13701. 


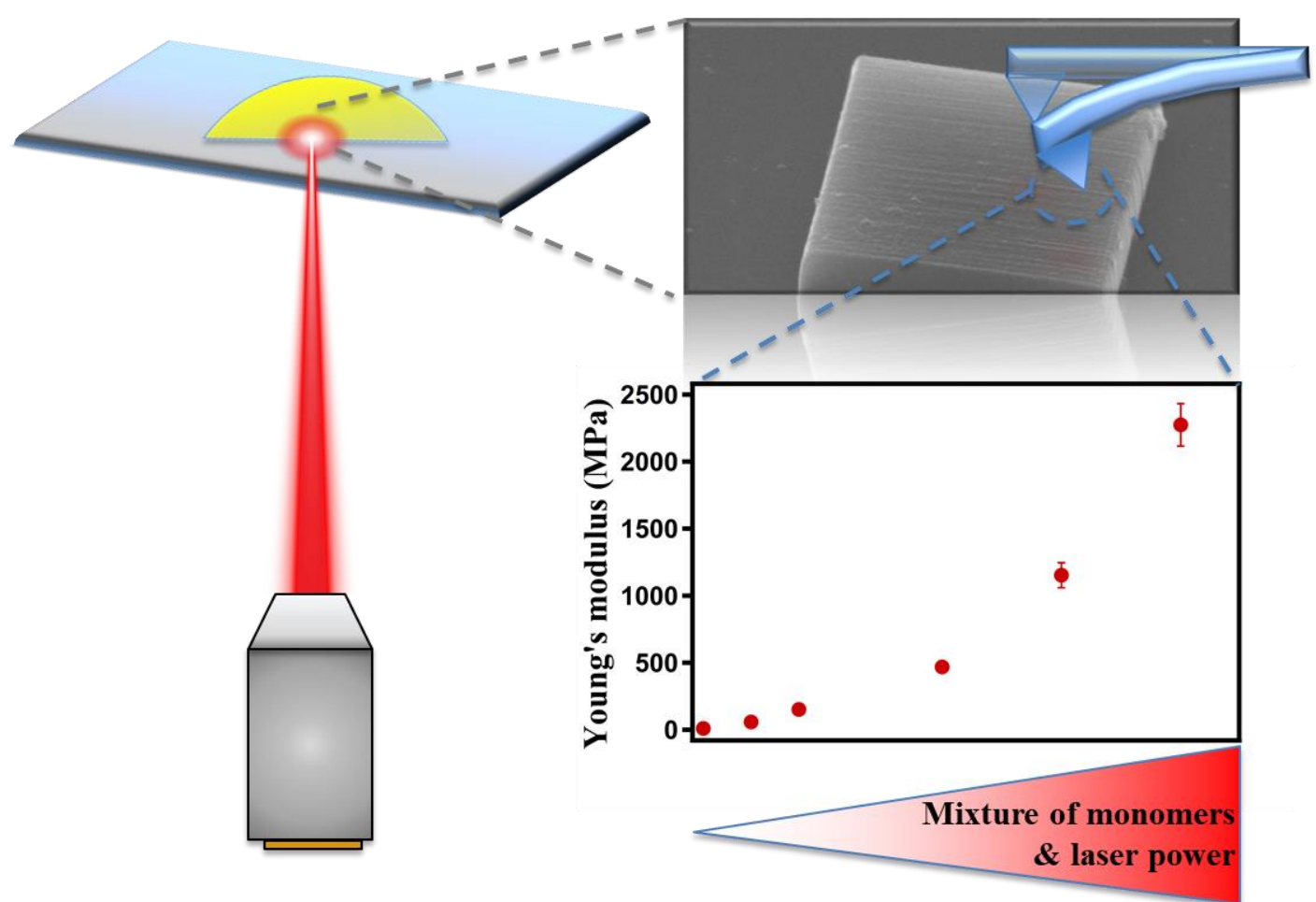




\section{Tuning nanomechanical properties of}

\section{microstructures made by 3D direct laser writing}

Mehdi Belqat ${ }^{1,2}$, Xingyu Wu ${ }^{1,2}$, Laura Piedad Chia Gomez ${ }^{1,2}$, Jean-Pierre Malval ${ }^{1,2}$, Sébastien 
Table S1 : Studied parameters and their typical used values

540 Table S2 : Average laser powers and the respective peak irradiance

\section{$541 \quad 2$. Roughness analysis}

542 Table S3 : RMS roughness and Young's modulus of PETA in function of average laser power.

Figure S1 : Topography image (left) and Young's modulus image (right) of PETA fabricated at an average laser power of $\mathrm{P}=6.73 \mathrm{~mW}$. $\mathrm{RMS}=36.36 \mathrm{~nm}$ and $\mathrm{E}=1.84 \mathrm{GPa}$.

545 Table S4 : RMS roughness and Young's modulus of TMPTA in function of average laser power.

546 Figure S2 : Topography image (left) and Young's modulus image (right) of TMPTA fabricated at an average laser power of $\mathrm{P}=9.93 \mathrm{~mW}$. RMS $=51.65 \mathrm{~nm}$ and $\mathrm{E}=1.67 \mathrm{GPa}$.

Table S5 : RMS roughness and Young's modulus of PEGDA in function of average laser power.

549 Figure S3 : Topography image (left) and Young's modulus image (right) of PEGDA fabricated at an average laser power of $\mathrm{P}=9.93 \mathrm{~mW}$. RMS $=27.1 \mathrm{~nm}$ and $\mathrm{E}=6.92 \mathrm{MPa}$.

551 Table S6: RMS roughness and Young's modulus of different mixtures of PETA and PEGDA

552 Figure S4: Topography image (left) and Young's modulus image (right) of 10\%PETA-90\%PEGDA 553 fabricated at an average laser power of $\mathrm{P}=13.5 \mathrm{~mW}$. RMS $=26.48 \mathrm{~nm}$ and $\mathrm{E}=57.6 \mathrm{MPa}$.

554 Figure S5 : Topography image (left) and Young's modulus image (right) of 20\%PETA-80\%PEGDA fabricated at an average laser power of $\mathrm{P}=13.5 \mathrm{~mW} . \mathrm{RMS}=36.89 \mathrm{~nm}$ and $\mathrm{E}=151.4 \mathrm{MPa}$.

Figure S6 : Topography image (left) and Young's modulus image (right) of 50\%PETA-50\%PEGDA fabricated at an average laser power of $\mathrm{P}=13.5 \mathrm{~mW}$. RMS $=36.94 \mathrm{~nm}$ and $\mathrm{E}=468 \mathrm{MPa}$.

558 Figure S7: Topography image (left) and Young's modulus image (right) of 75\%PETA-25\%PEGDA

\section{3. Morphology characterizations}

561 Figure S8 : SEM images of 2D PETA structures made by 3D DLW at A) $6.73 \mathrm{~mW}$, B) $11.36 \mathrm{~mW}$, C) $56213.5 \mathrm{~mW}$

563 Figure S9 : SEM images of 2D PEGDA structures made by 3D DLW at A) $9.92 \mathrm{~mW}$, B) 10.92, C) 13.5 $564 \mathrm{~mW}$.

565 Figure S10 : SEM images of 2D structures made by 3D DLW from mixture of PETA and PEGDA: A) 566 10\%PETA-90\%PEGDA

B) $20 \%$ PETA-80\%PEGDA,

C) $50 \%$ PETA-50\%PEGDA,

D) $75 \%$ PETA$25 \%$ PEGDA.

4. Determination of degree of conversion by FTIR microspectroscopy

Figure S11 : FTIR spectra of resins with different weight percentages of PETA and PEGDA (blue lines) and the corresponding two-photon polymerized squares (red lines, laser power $13.5 \mathrm{~mW}$ was used for all squares). 
574 Table S1 : Studied parameters and their typical used values

\begin{tabular}{|c|c|}
\hline Parameter & Value \\
\hline Wavelength $\lambda^{1}$ & $800 \mathrm{~nm}$ \\
Repetition rate $f^{1}$ & $80 \mathrm{MHz}$ \\
Pulse duration $\tau^{1}$ & $140 \mathrm{fs}$ \\
Numerical aperture $N A^{1}$ & 0.65 \\
Lateral dimension $\mathrm{d}_{l}{ }^{1}$ & $1.5 \mu \mathrm{m}$ \\
Exposure time $t_{\text {exp }}{ }^{1}$ & $10 \mathrm{~ms}$ \\
Slicing $\Delta^{1}$ & $300 \mathrm{~nm}$ \\
Pulse Energy $E$ & $84.1 \mathrm{pJ}-168.8 \mathrm{pJ}$ \\
Average laser power $P_{a}$ & $6.73-13.5 \mathrm{~mW}$ \\
Peak irradiance $I^{2}$ & $0.009-0.019 \mathrm{TW} / \mathrm{cm}^{2}$ \\
\hline
\end{tabular}

\section{${ }^{1}$ N.B.: all these parameters are kept constant for the current study. Only the average laser power is tuned.}

${ }^{2}$ N.B.: the peak irradiance I is sometimes referred to as "intensity".

Lateral dimension, pulse energy and peak irradiance are calculated according to equations (1), (2) and (3) respectively. Lateral dimension of the voxel is calculated thanks to the equation:

$$
d_{l}=\frac{1.22 \times \lambda}{N A}
$$

where $\lambda$ is the excitation wavelength, $N A$ the numerical aperture of the objective. Pulse energy E is then calculated using equation (2) :

$$
E=\frac{P_{a}}{f}
$$

where $P_{a}$ is the average power in $\mathrm{W}$ and $\mathrm{f}$ the repetition rate in $\mathrm{Hz}$. Peak irradiance is finally obtained thanks to the following equation :

$$
I=\frac{2 E}{\pi \cdot d_{l}^{2} \cdot \tau \cdot \sqrt{\pi}}(3)
$$

where $\tau$ is the pulse duration.

Table S2 : Average laser powers and the respective peak irradiance

\begin{tabular}{|c|c|}
\hline Average laser power $(\mathbf{m W})$ & Peak irradiance $\left(\mathbf{T W} / \mathbf{c m}^{\mathbf{2}}\right)$ \\
\hline 6.73 & $9.59 \times 10^{-3}$ \\
9.92 & $14.1 \times 10^{-3}$ \\
11.36 & $16.1 \times 10^{-3}$ \\
12.03 & $17.1 \times 10^{-3}$ \\
12.8 & $18.2 \times 10^{-3}$ \\
13.5 & $19.2 \times 10^{-3}$ \\
\hline
\end{tabular}


2. Roughness analysis: The root mean square (RMS) roughness was used to measure the surface roughness of the several samples fabricated during this study. The RMS roughness and Young's modulus obtained for each monomers at different average laser powers are presented in Tables S3, S4 and S5 corresponding to PETA, TMPTA and PEGDA respectively. Besides, topography and Young's modulus images obtained by AFM are presented for the 3 monomers at the energy threshold (Figure S1 to S3), as well as for two mixtures (20 and 50\% PETA, Figure S4 and S5).

Table S3 : RMS roughness and Young's modulus of PETA in function of average laser power.

\begin{tabular}{|c|c|c|c|}
\hline $\begin{array}{c}\text { Average laser } \\
\text { power }(\mathbf{m W})\end{array}$ & RMS roughness (nm) & Young's Modulus (GPa) & Stdev (GPa) \\
\hline $\mathbf{6 . 7 3}$ & 39.36 & 1.843 & 0.08 \\
\hline $\mathbf{9 . 9 2}$ & 44.31 & 1.93 & 0.07 \\
\hline $\mathbf{1 1 . 3 6}$ & 34.94 & 2.062 & 0.08 \\
\hline $\mathbf{1 2 . 0 8}$ & 39.26 & 2.15 & 0.06 \\
\hline $\mathbf{1 3 . 5}$ & 48.61 & 2.274 & 0.16 \\
\hline
\end{tabular}
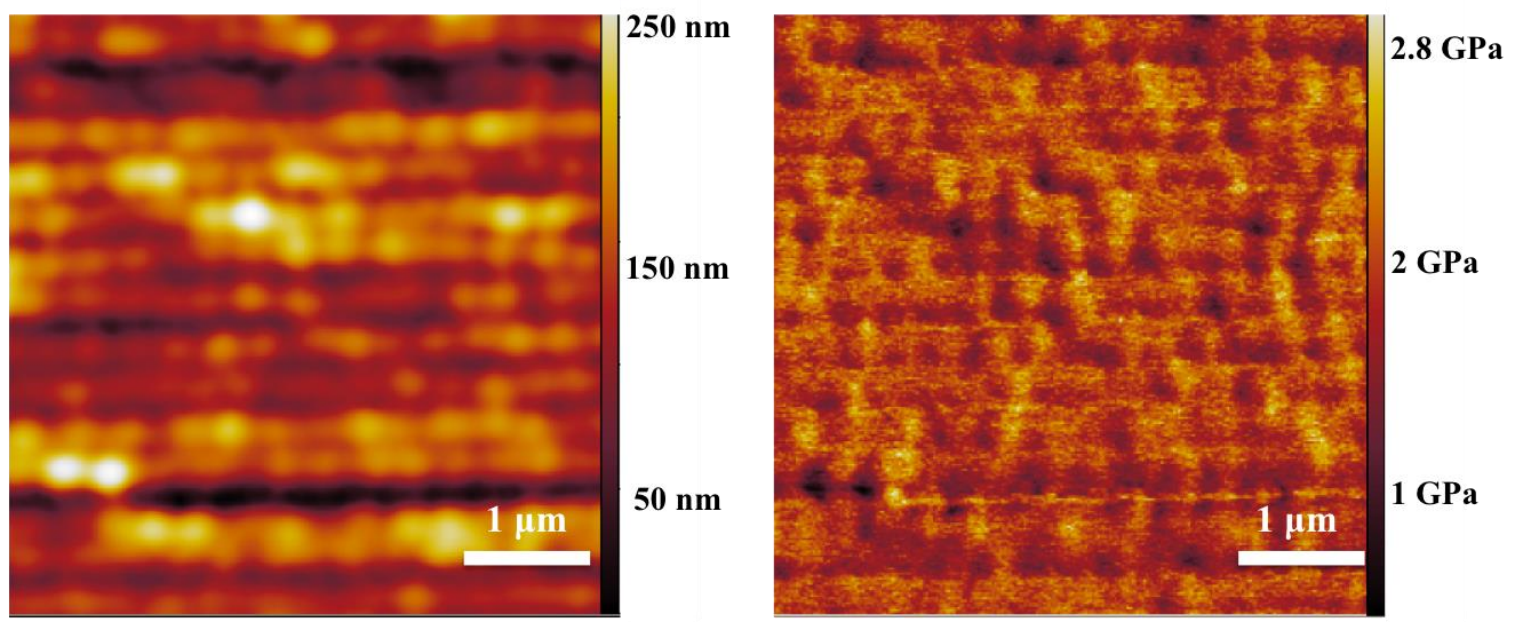

Figure S1 : Topography image (left) and Young's modulus image (right) of PETA fabricated at an average laser power of $\mathrm{P}=6.73 \mathrm{~mW}$. $\mathrm{RMS}=36.36 \mathrm{~nm}$ and $\mathrm{E}=1.84 \mathrm{GPa}$. 
Table S4 : RMS roughness and Young's modulus of TMPTA in function of average laser power.

\begin{tabular}{|c|c|c|c|}
\hline $\begin{array}{c}\text { Average laser } \\
\text { power }(\mathbf{m W})\end{array}$ & RMS roughness (nm) & Young's Modulus (GPa) & Stdev (GPa) \\
\hline $\mathbf{9 . 9 2}$ & 51.65 & 1.67 & 0.06 \\
\hline $\mathbf{1 1 . 3 6}$ & 50.83 & 1.77 & 0.07 \\
\hline $\mathbf{1 2 . 0 8}$ & 50.9 & 2.08 & 0.05 \\
\hline $\mathbf{1 3 . 5}$ & 47.7 & 2.3 & 0.11 \\
\hline
\end{tabular}
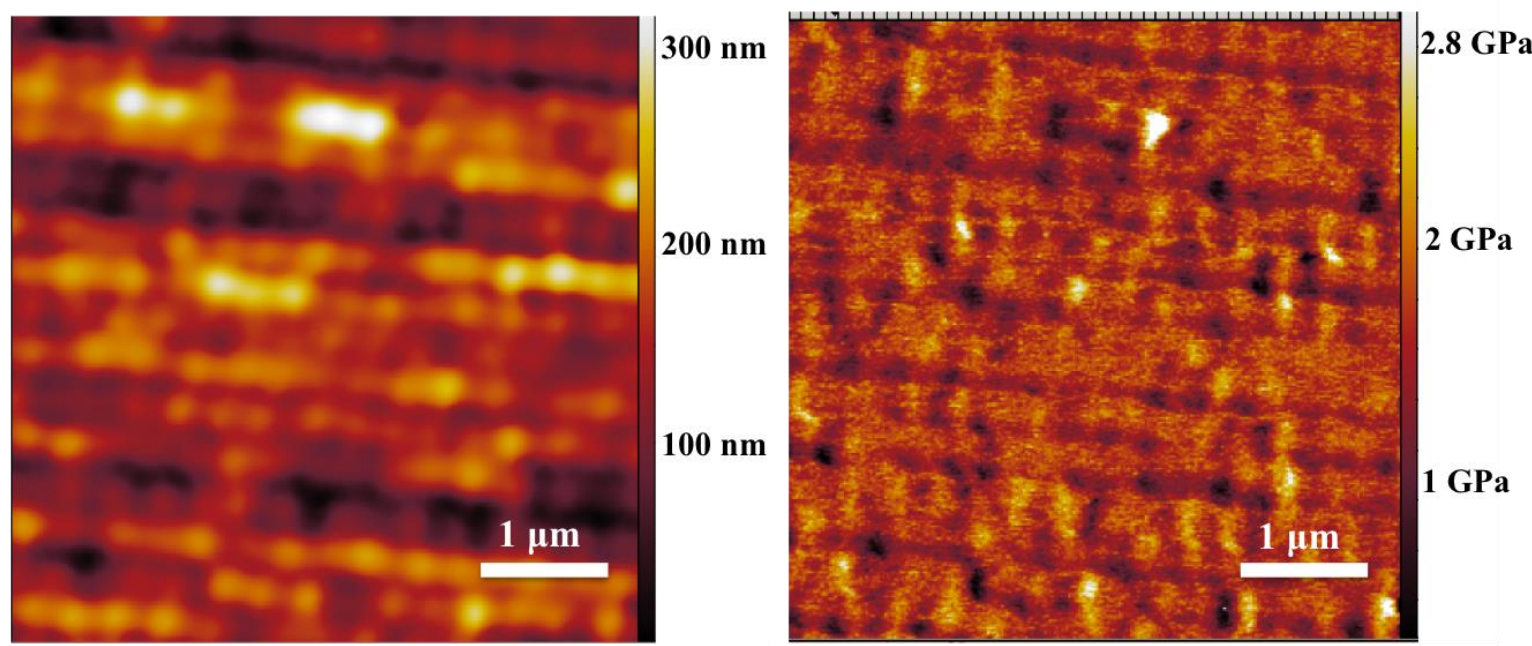

611 Figure S2 : Topography image (left) and Young's modulus image (right) of TMPTA fabricated at an 612 average laser power of $\mathrm{P}=9.93 \mathrm{~mW}$. $\mathrm{RMS}=51.65 \mathrm{~nm}$ and $\mathrm{E}=1.67 \mathrm{GPa}$.

614 Table S5 : RMS roughness and Young's modulus of PEGDA in function of average laser power.

\begin{tabular}{|c|c|c|c|}
\hline $\begin{array}{c}\text { Average laser } \\
\text { power (mW) }\end{array}$ & RMS roughness (nm) & Young's Modulus (MPa) & Stdev (MPa) \\
\hline $\mathbf{9 . 9 2}$ & 27.1 & 6.92 & 0.07 \\
\hline $\mathbf{1 1 . 3 6}$ & 20 & 8.21 & 0.08 \\
\hline $\mathbf{1 2 . 0 3}$ & 30.9 & 8.89 & 0.2 \\
\hline $\mathbf{1 2 . 8}$ & 28 & 9.14 & 0.2 \\
\hline $\mathbf{1 3 . 5}$ & 26.9 & 9.47 & 0.38 \\
\hline
\end{tabular}



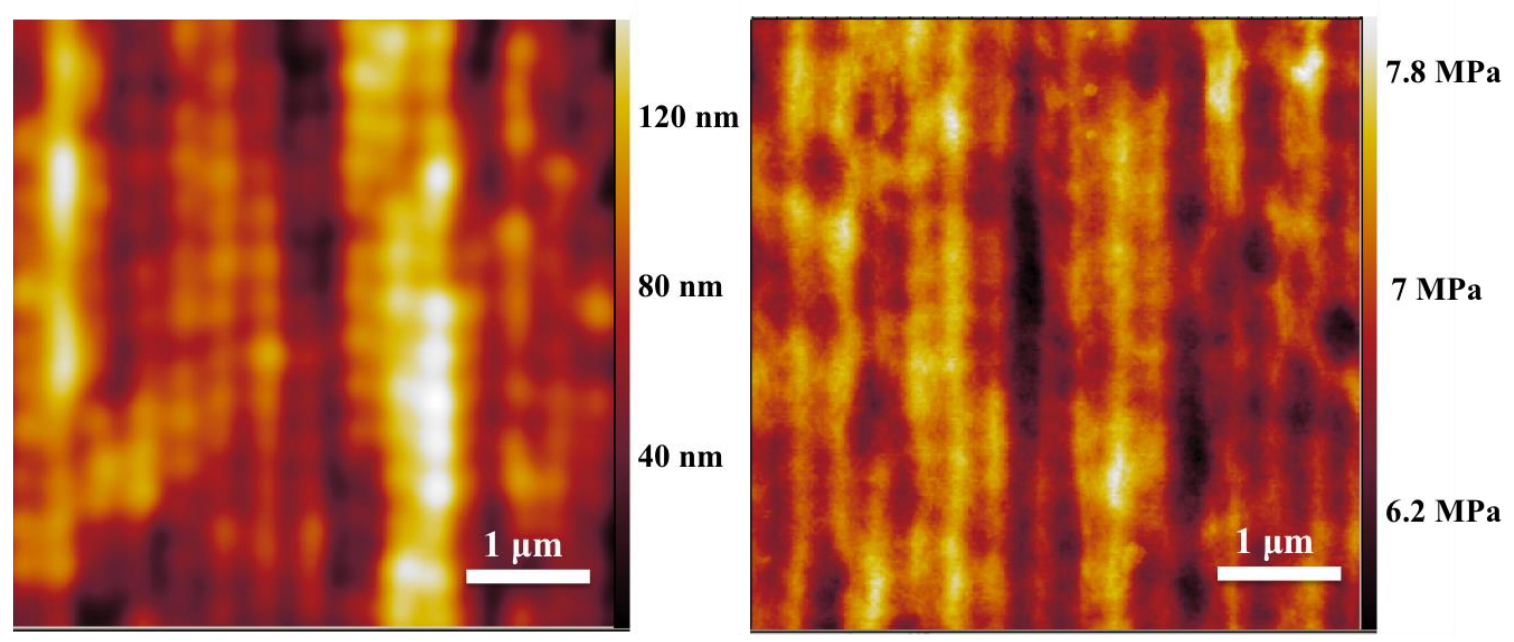

617 Figure S3 : Topography image (left) and Young's modulus image (right) of PEGDA fabricated at an average laser power of $\mathrm{P}=9.93 \mathrm{~mW}$. RMS $=27.1 \mathrm{~nm}$ and $\mathrm{E}=6.92 \mathrm{MPa}$.

Table S6: RMS roughness and Young's modulus of different mixtures of PETA and PEGDA.

\begin{tabular}{|c|c|c|c|}
\hline Mixture & $\begin{array}{c}\text { RMS roughness } \\
(\mathbf{n m})\end{array}$ & Young's Modulus (MPa) & Stdev (MPa) \\
\hline 10\% PETA - 90\% PEGDA & 26.48 & 57.6 & 4.3 \\
\hline 20\%PETA - 80\% PEGDA & 36.89 & 151.4 & 9 \\
\hline 50\%PETA - 50\% PEGDA & 36.94 & 468 & 93.9 \\
\hline 75\%PETA - 25\% PEGDA & 45.34 & 1153 & 9.7 \\
\hline
\end{tabular}
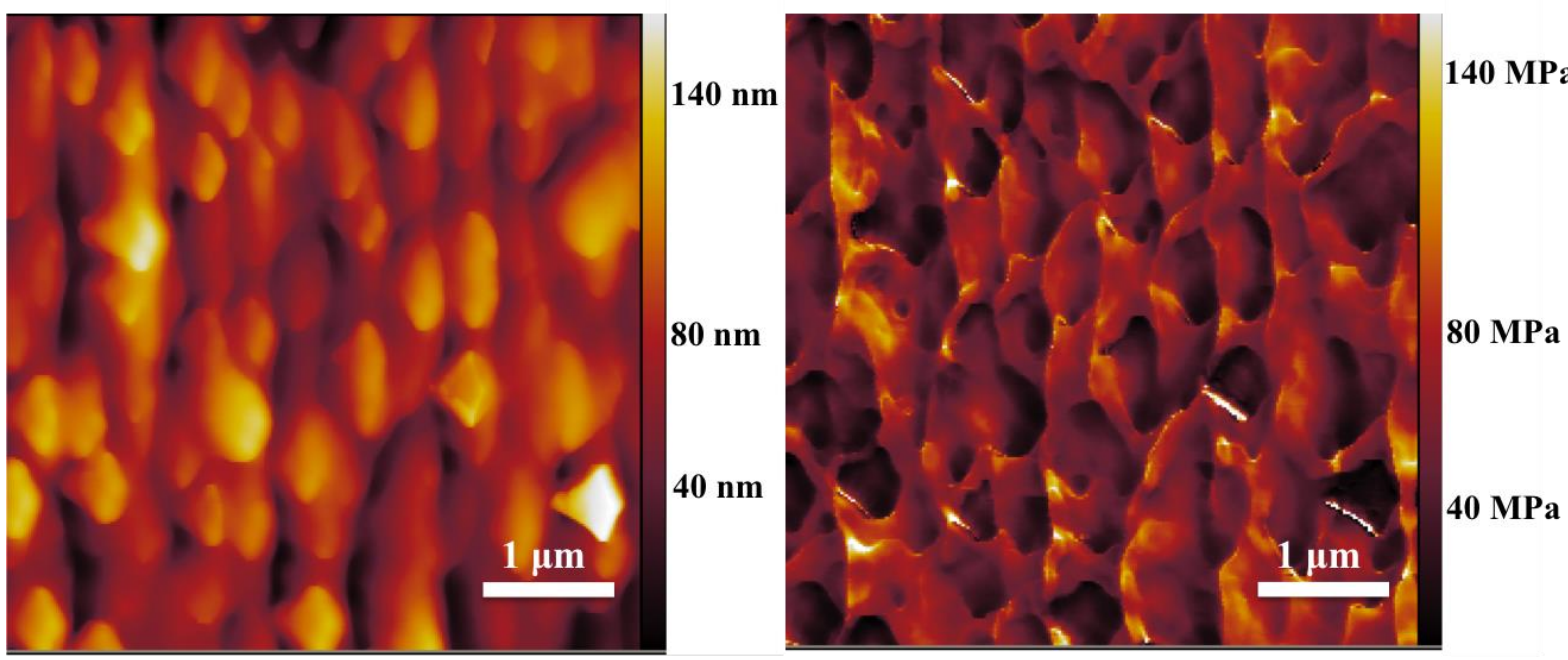

Figure S4: Topography image (left) and Young's modulus image (right) of 10\%PETA-90\%PEGDA 624 fabricated at an average laser power of $\mathrm{P}=13.5 \mathrm{~mW}$. RMS $=26.48 \mathrm{~nm}$ and $\mathrm{E}=57.6 \mathrm{MPa}$. 


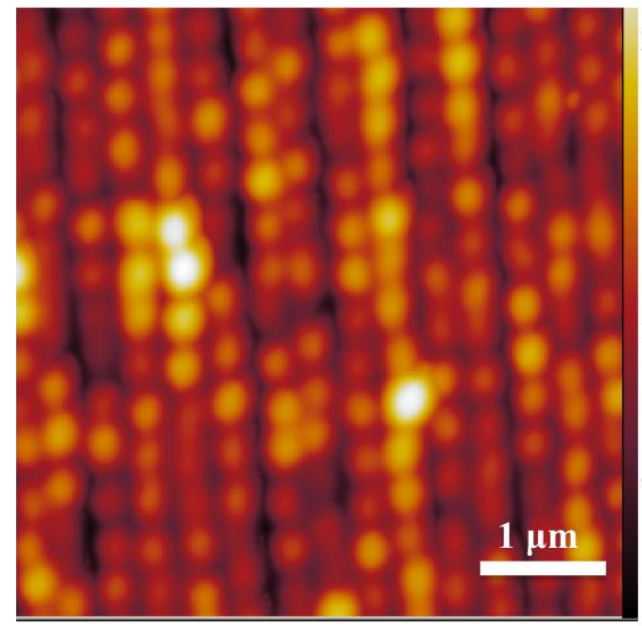

$240 \mathrm{~nm}$

Figure S5 : Topography image (left) and Young's modulus image (right) of 20\%PETA-80\%PEGDA 627

fabricated at an average laser power of $\mathrm{P}=13.5 \mathrm{~mW}$. RMS $=36.89 \mathrm{~nm}$ and $\mathrm{E}=151.4 \mathrm{MPa}$.
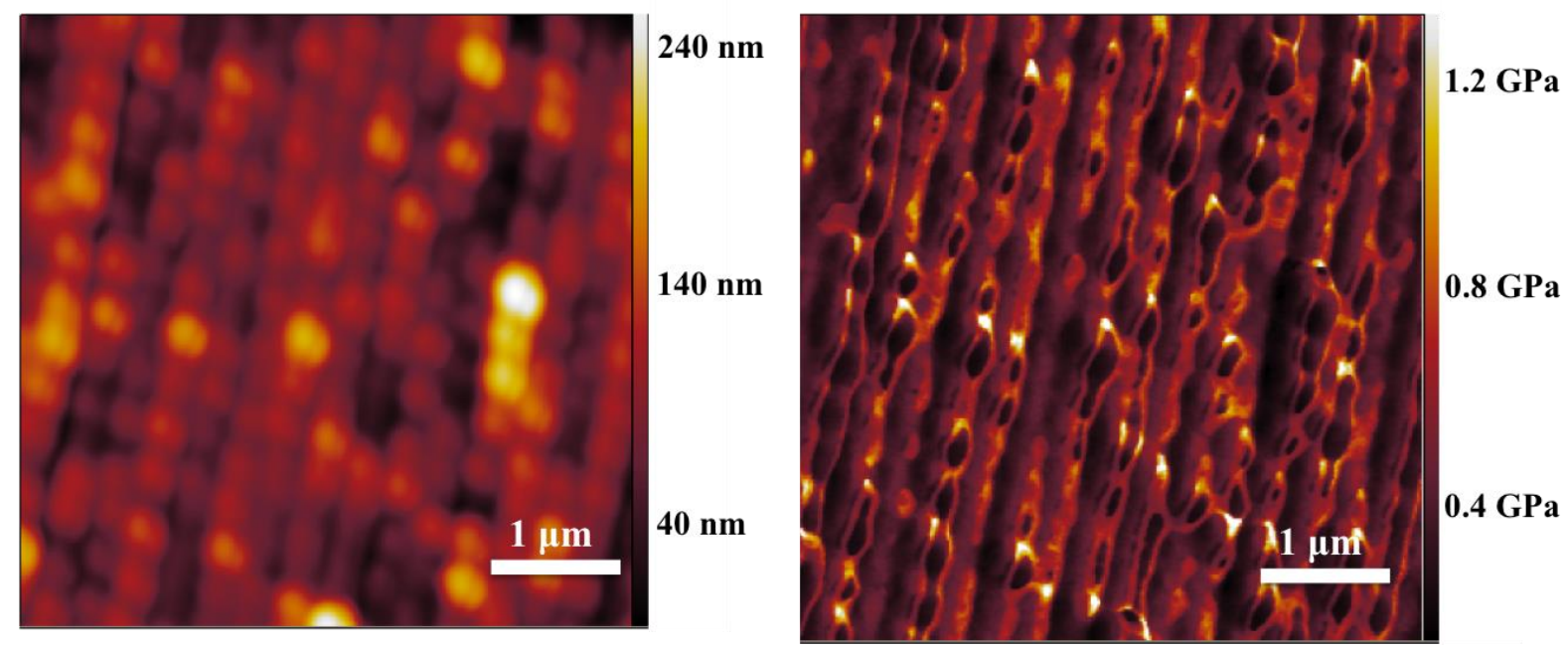

Figure S6 : Topography image (left) and Young's modulus image (right) of 50\%PETA-50\%PEGDA
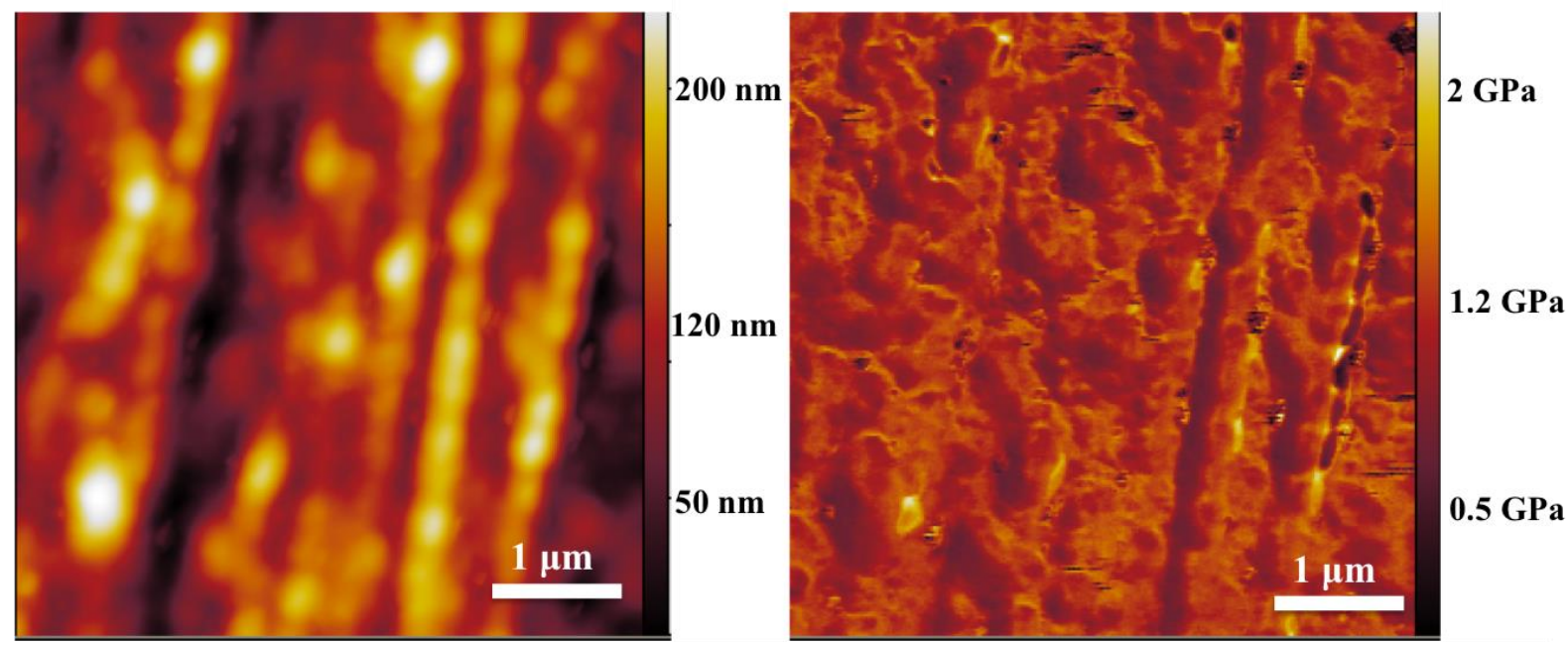

Figure S7: Topography image (left) and Young's modulus image (right) of 75\%PETA-25\%PEGDA 
3. Morphology characterization by scanning electron microscopy (SEM):
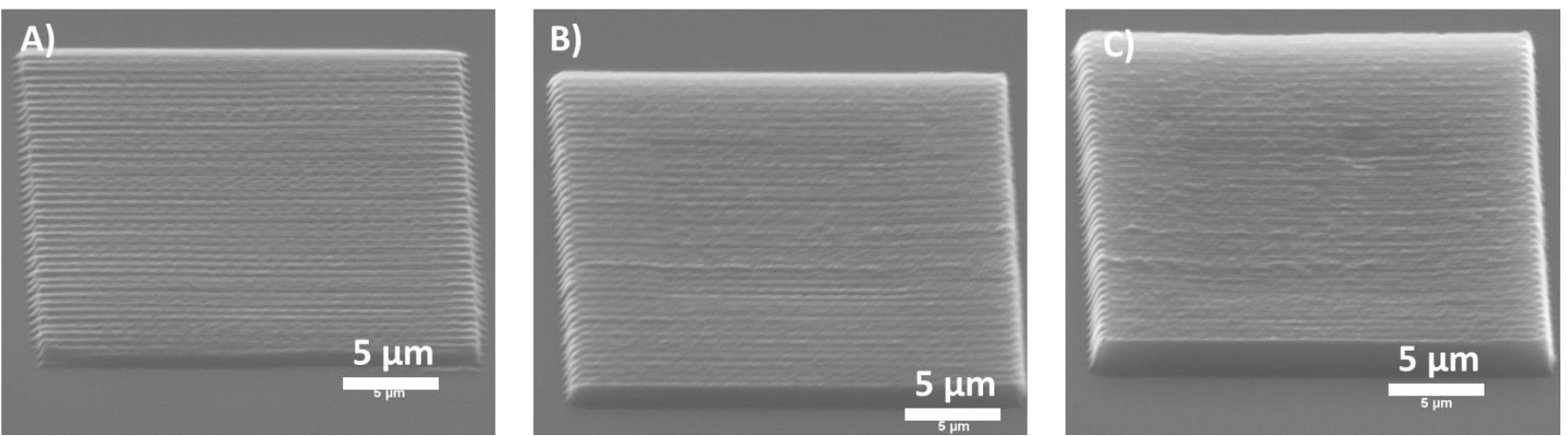

Figure S8 : SEM images of 2D PETA structures made by 3D DLW at A) $6.73 \mathrm{~mW}, \mathrm{~B}) 11.36 \mathrm{~mW}, \mathrm{C})$ $13.5 \mathrm{~mW}$.

\section{A)}

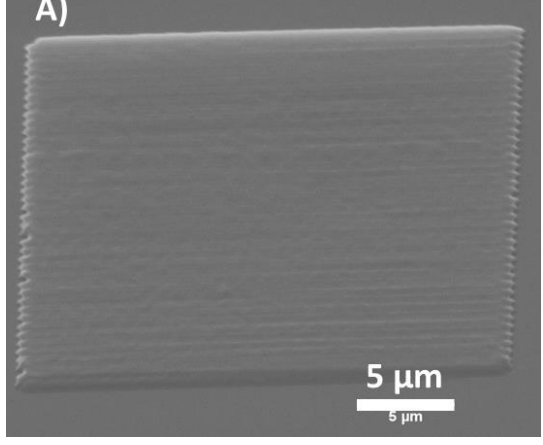

B)

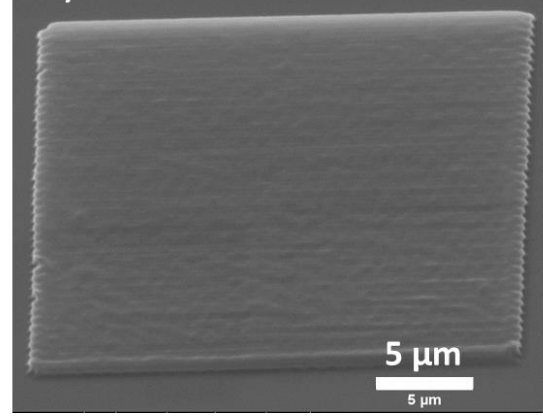

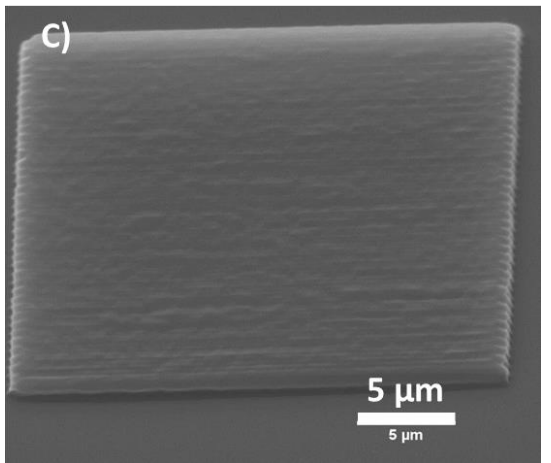

Figure S9 : SEM images of 2D PEGDA structures made by 3D DLW at A) $9.92 \mathrm{~mW}$, B) 10.92 , C) 13.5 $\mathrm{mW}$.
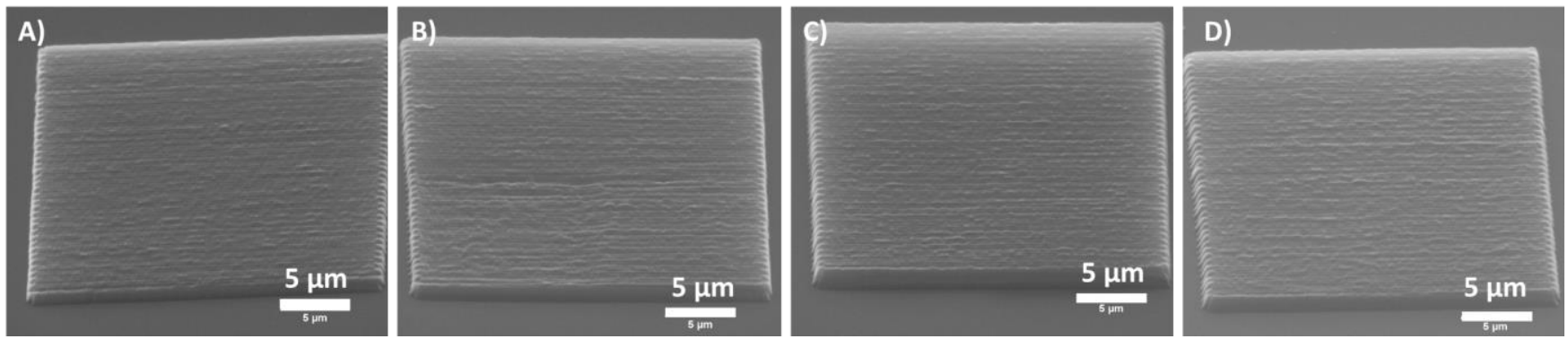

641 Figure S10 : SEM images of 2D structures made by 3D DLW from mixture of PETA and PEGDA: A) 642 10\%PETA-90\%PEGDA，B) 20\%PETA-80\%PEGDA，C) 50\%PETA-50\%PEGDA， D) 75\%PETA643 25\%PEGDA.

\section{Determination of the degree of conversion (DC) by ATR FTIR microspectroscopy :}

646 Micro-FTIR (Fourier-Transform Infrared spectroscopy) tests were performed with a Thermo Scientific 647 Nicolet iN10 MX on polymers squares of 20 micrometers length using a germanium tip ATR. The 648 analyzed zone was 5 micrometers at the center of the squares. The absorbance spectra were measured 649 from $4000 \mathrm{~cm}^{-1}$ to $675 \mathrm{~cm}^{-1}$ wavenumber with a resolution of $8 \mathrm{~cm}^{-1}$ and 64 scans per spectrum. This 650 method relies on measuring the degree of conversion (DC) of the structured polymers in comparison 651 with the resin mixture. The determination of the DC allow to quantify the amount of consumed carbon- 
carbon double bonds $(\mathrm{C}=\mathrm{C})$ during the photopolymerization. It is determined using the following equation from [1]:

$$
D C=\left[1-\frac{A_{C=C} / A_{C=O}}{A^{\prime} C=C / A^{\prime} C=O}\right] \times 100
$$

with $\mathrm{A}_{\mathrm{C}=\mathrm{C}}, \mathrm{A}_{\mathrm{C}=\mathrm{O}}, \mathrm{A}^{\prime}{ }_{\mathrm{C}=\mathrm{C}}$ and $\mathrm{A}^{\prime}{ }_{\mathrm{C}=\mathrm{O}}$ the integrated area of absorption bands related to the $\mathrm{C}=\mathrm{C}$ and $\mathrm{C}=\mathrm{O}$ moieties in the polymerized and the nonpolymerized resin, respectively.

After irradiation

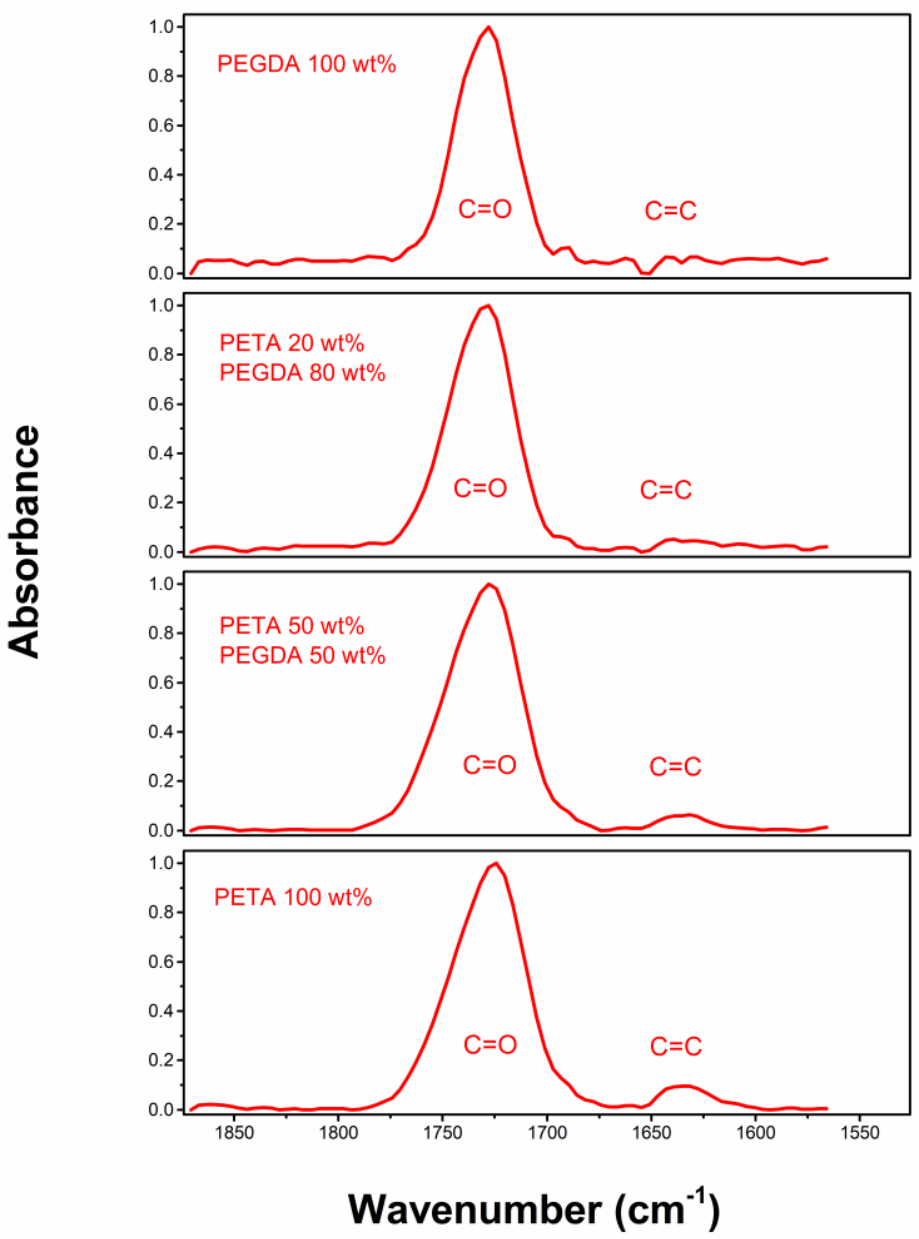

Before irradiation
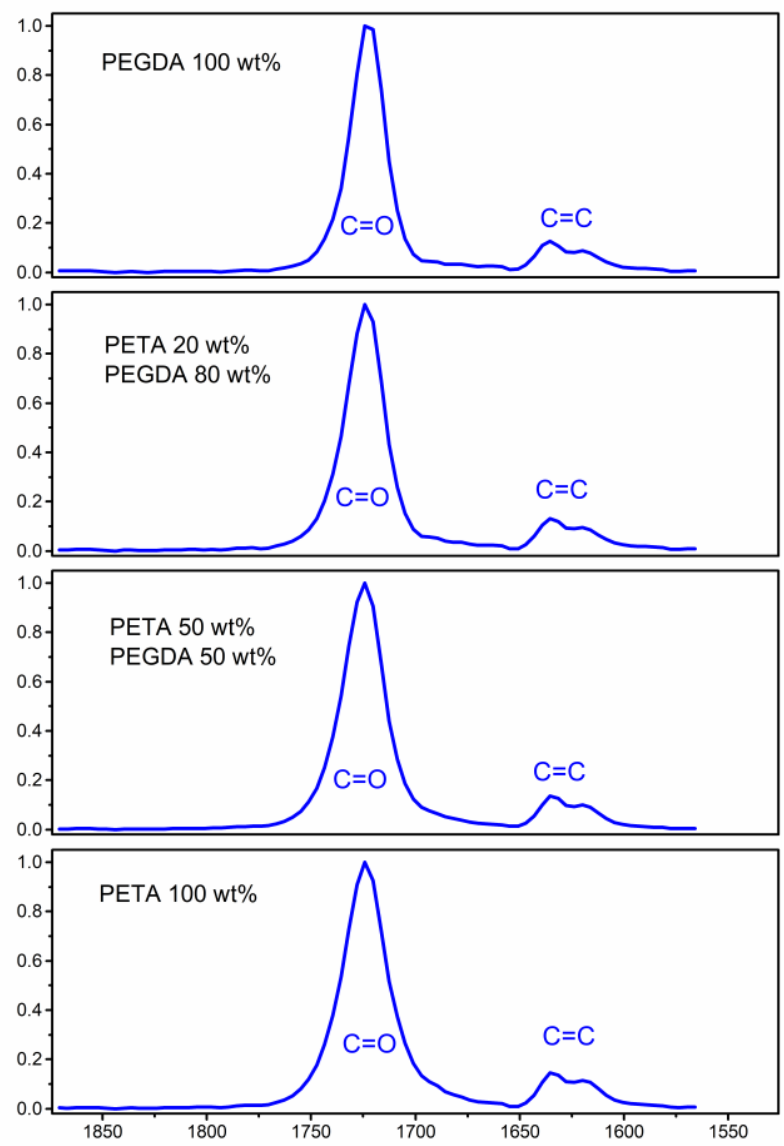

Wavenumber $\left(\mathrm{cm}^{-1}\right)$

Figure S11 : FTIR spectra of resins with different weight percentages of PETA and PEGDA (blue lines) and the corresponding two-photon polymerized squares (red lines, laser power $13.5 \mathrm{~mW}$ was used for all squares).

References: 